\title{
Longitudinal Aging Study in India (LASI): new data resources for addressing aging in India
}

\author{
The Longitudinal Aging Study in India (LASI), the largest national health and retirement study in the world, released \\ its wave 1 microdata earlier this year. The principal investigators of LASI introduce the study and explain how it can \\ advance aging research in India and beyond in response to the impending challenges of rapid population aging.
}

\author{
David E. Bloom, T. V. Sekher and Jinkook Lee
}

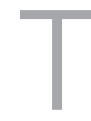
he older population of India is currently the world's second-largest: 140 million people aged 60 and above (compared to 250 million people in China) ${ }^{1}$. Moreover, the average annual growth rate of the older population is almost three times higher than the overall population growth rate of India ${ }^{2}$. The underlying population dynamics foretell that the proportion of India's population aged 60 and above will more than double, from $9 \%$ in 2015 to $19 \%$ in 2050 (ref. ${ }^{1}$ ); this will result in an older population of more than 320 million, almost equal to the entire (current) population of the USA.

Older population shares are rapidly rising throughout the world owing to increasing life expectancy, declining fertility and large-sized cohorts reaching the older ages. These demographic shifts led the World Health Organization and the United Nations to declare 2021-2030 as the 'Decade of Healthy Ageing', an initiative of research, policy development and outreach that is designed to understand and communicate the experience of aging and the needs of aging populations in the public health ecosystem ${ }^{3}$. Aging poses particular health, social and economic challenges for Indian policymakers owing to the size, growth and structure of India's population. Health challenges take the form of increasing prevalence of noncommunicable diseases, including cardiovascular disease, cancer, respiratory diseases, diabetes and mental health issues; these challenges also manifest as increasing physical and functional disabilities, including issues related to eyesight and hearing. In fact, underdiagnosed diseases such as hypertension and diabetes (which are growing due to the lack of health literacy and weak health-seeking behaviors), along with a lack of quality and accessible healthcare services, will only intensify the health-related challenges of population aging. Social challenges entail issues related to who, how and what care - inclusive of physical and emotional care and companionship - will be needed by older people. Economic challenges involve the expected decline in labor force participation rates and the fact that older people consume more than they produce, which would further strain an Indian economy that will already be scrambling to pay for the additional healthcare needs of an older population. These challenges are further compounded by systemic and behavioral inequities, in terms of, for example, gender, religion, caste, education, state-level health systems and urban-rural residence.

Population aging in India is inevitable, and the country is currently ill-prepared to deal with the expanding and evolving needs of older adults. This demographic phenomenon will have important policy implications, to which the country must start adapting. Currently, India has a familybased culture, in which family members are typically expected to be responsible for taking care of their parents or grandparents. However, changing modern social structure, which is drifting away from the extended joint family system to nuclear families (that is, parents and their dependent children), coupled with migration to urban areas and to other countries will greatly stress the existing system, and proactive interventions by the government will be necessary. Governments (both national and state, the latter of which currently sets the majority of health policies) might have to bear some or all of the responsibilities of providing economic security, social wellbeing, geriatric healthcare services and long-term care to this important population. Many countries have a higher share of older population than India, and most of these countries have models that may be transported or adapted to the context of India. Given the scale of the impending demographic shift, it is also possible that India will need an altogether new model to care for older adults. The Government of India is well aware of these looming challenges and their policy implications, which is why it is a strong supporter of collecting publicly available aging-related data. In January 2021, the Ministry of Health and Family Welfare, Government of India, released the national report for wave 1 of the Longitudinal Aging Study in India (LASI). LASI was initially conceptualized in 2006, with its first pilot being conducted in 2010-2011. In 2013, the first two waves of LASI were funded predominantly by the National Institute on Aging (USA) and the Ministry of Health and Family Welfare (Government of India). LASI is led by a team of principal investigators that encompasses health, economic, social, and data collection expertise, coupled with research experience in India.

\section{Characterizing LASI and its position}

LASI provides high-quality, scientific and comprehensive data on the health, social and economic aspects of aging in India. The first wave of LASI (2017-2019) enrolled more than 72,000 individuals aged 45 and older, along with their spouses (irrespective of age), making it the largest health and retirement study in the world. By comparison, the second-largest health and retirement study began in the USA in 1992 and interviewed 20,912 individuals in its latest wave. The LASI survey has achieved an impressive response rate at both the household and individual levels: the overall household response rate was $96 \%$ and the individual response rate was $87 \%{ }^{4}$. This individual response rate is comparable to other health and retirement studies $-87 \%$ for wave 13 (2014) of the USA's health and retirement study ${ }^{5}$ and $86 \%$ for wave 4 (2018) of the China Health and Retirement Longitudinal Study ${ }^{6}$. The LASI wave 1 sample is representative at the national and state and union territory levels and is also reasonably harmonized (to the extent it is cross-nationally appropriate), with the survey instruments being fielded in health and retirement studies that are ongoing in 46 other countries around the 


\section{Box 1 | Policy implications drawn from LASI wave 1 data}

- Design and implement targeted noncommunicable disease prevention and management programs, including those targeted toward 'brain health'.

- Assess the need for disease prevention programs (for example, adult vaccination, tobacco and alcohol use awareness, and physical activity).

- Strengthen geriatric care in India.

- Adapt medical curriculum to expand and integrate geriatric medicine (focus on assessing, treating and managing multimorbidities).

- Expand the number and capacity of geriatric centers.

- Integrate geriatric care in primary, secondary and tertiary healthcare services, including community-based health services.

- Assess the value of social welfare and government health programs and innovate on their design and implementation, with particular focus on vulnerable, abused and neglected geriatric populations.

- Assess the need for and value of agefriendly infrastructure and housing.

- Conduct cross-state comparisons to determine best practices (for example, long-term care, home-based care, legal protection and healthcare spending).
- Assess the economic vulnerability of the older population and design pension, health, long-term care and other old-age programs in response.

- Develop retirement preparedness programs, as well as programs targeted toward enhancing the health and financial literacy among the general population, particularly among older adults.

- Develop national dementia prevention strategies to reduce dementia risk at the population level, especially targeting at-risk subpopulations.

- Build national and subnational government programs to meet the challenges of caring for persons living with dementia and their care givers.

- Inform the Government of India's COVID-19 pandemic response. The government will have more information on comorbidities, helping them to identify the communities in the greatest need and thereby plan moreefficient vaccine distribution. To collect even more detailed data about the effects of the pandemic on the older population in India, we plan to include a supplemental questionnaire on COVID-19 in the forthcoming LASI wave 2 .

world, facilitating the conduct of meaningful cross-state and cross-national comparisons (see 'Concordance across Surveys' at Gateway to Global Aging Data (https:// g2aging.org/concordance) for detailed concordance information). Enabling cross-state comparisons is especially important in India, as health policy is a state responsibility in the country, and because there exists considerable socioeconomic, institutional and demographic diversity across the states. On the other hand, crossnational comparisons will help India and other countries to learn from each other's experiences and to build more robust models of care for older adults.

LASI's household modules include detailed questions on household members, their housing and built environment, as well as household consumption, assets, debts, income and insurance. LASI's individual modules include detailed questions on respondents' demographics, health status, healthcare access and use, health behaviors, family and social network, social security programs, work, retirement, pension, and experimental modules on time use, social connectedness, and expectations about their health, work and survival. The individual modules also include physical biomarkers related to visual acuity, anthropometric measurements, lung function, gait and blood pressure. The collection of dried blood spots is another unique asset of LASI, which can be used to support genomic sequencing and can be assayed to provide biologic measures of health conditions that can then be correlated with the phenotypic data collected through the survey.

LASI therefore offers detailed information on a host of understudied variables, including chronic health conditions, disability and assistance, life and family history, social and economic wellbeing, and participation in old age benefit schemes. Successive waves of LASI, which are planned every three years, will involve overlapping but non-identical instruments (for example, we anticipate wave 2 will include two additional modules, a life history module and a COVID-19 module). The longitudinal nature of LASI will allow researchers to assess developments and changes in the life of older adults as they transition to older ages. It will also enable the study of how different interventions (as state-level policies and programs are introduced) affect the experience of aging. Finally, from a technical standpoint, LASI used computer-assisted personal interviewing (which facilitates real-time data-quality checks), contributing to the quality of LASI data.

Another distinguishing feature of LASI is that it attaches an allied study that focuses on late-life cognition and dementia $\left(\right.$ LASI-DAD) ${ }^{7}$ in India. The first wave of LASI-DAD, which spanned from October 2017 to the end of March 2020, recruited a nationally representative subsample of more than 4,000 LASI respondents aged 60 or older from 18 states and union territories across the country. Alzheimer's disease and related dementias (ADRDs) contributed 33.1 million disability-adjusted life years - a common measure of overall disease burden expressed as the number of years lost owing to illness, disability or death - worldwide in $2019^{8}$. A recent estimate predicts that ADRDs will contribute 115.8 million disability-adjusted life years in 2050, which means the global burden of ADRDs will more than triple in the coming 30 years $^{8}$. ADRDs currently have no cure and lack highly effective therapeutic interventions. In addition to the enormous health toll affecting people with dementia and their families, ADRDs extract a massive economic toll - one that is shifting to low- and middle-income countries, as their populations are aging relatively quickly. A recent study estimated that the global costs for dementia were US $\$ 957.56$ billion in 2015 , a figure that is projected to balloon to US $\$ 9.12$ trillion by $2050^{\circ}$. Boosting research and investments into ADRDs is fundamental to fight this surge, and we hope the release of the LASI-DAD microdata will have a role in encouraging these necessary endeavors.

\section{Accessing LASI data}

The LASI wave 1 microdata and related documentation are freely available at https:// lasi-india.org and https://iipsindiaac.in/ content/LASI-data. LASI-DAD wave 1 data can be accessed at https://lasi-dad.org.

\section{Key findings about aging in India from LASI wave 1 data}

So, what do the data say about the experience of aging in India? Unsurprisingly, the LASI data - as presented in the 2017-2018 national report ${ }^{4}$ and other published articles $^{10-14}$ - confirm that the prevalence of chronic diseases increases with age, with a higher prevalence among older adults (aged 60 years and above) compared to those in the 45-59-yearold age group. There is a prevalence of undiagnosed and underdiagnosed diseases, 
such as hypertension, diabetes and vision impairment. Noncommunicable diseases are the major cause of hospitalization among older adults, yet health insurance coverage among this cohort is only around $20 \%$. Gender differences in the health, social and economic wellbeing of older adults are prominent; some diseases - such as heart disease, stroke, diabetes mellitus, chronic lung disease and neurological problems - are higher among men, while women are more likely to be diagnosed with hypertension, anemia, bronchitis, depression, Alzheimer's disease and dementia, any bone or joint disease, and cancer. The data reveal other disparities as well: place of residence, caste, religion, education and income have a considerable effect on the wellbeing of older adults. A large proportion of older adults are working in the informal sector and, thus, ineligible to receive a pension when they retire or stop working, which exacerbates the problem of consumption outpacing production for this group. In a telling snapshot of the situation, only around $50 \%$ of older adults are 'highly satisfied' with their lives. Using the data from LASI-DAD, the prevalence of dementia at the national level was estimated as $7.4 \%{ }^{15}$. These new epidemiological data provide an unprecedented opportunity to study cognitive aging and its risk factors in India ${ }^{16}$.

The public release of the LASI and the LASI-DAD data, which can be accessed by interested users free of cost, is creating numerous opportunities to conduct novel high-quality research throughout India and beyond to analyze the data and better understand the aging experience. It is our hope that these data lead to the design of policies to improve that experience and the prospects for healthy aging. Examples of policy implications are outlined in Box 1. These policies will require considerable resources, coordination and commitment across sectors and stakeholders to bring this aspiration to fruition. These resources, however, are an investment in a superior future: a future with a healthier, happier, more economically and socially secure, and a more independent older population. While population aging in India and across the globe is inevitable, its consequences are not.

\section{David E. Bloom (DD 1 , T. V. Sekher ${ }^{2}$ and Jinkook Lee (D) ${ }^{3}$}

${ }^{1}$ Harvard T. H. Chan School of Public Health, Boston, MA, USA. International Institute for Population Sciences, Mumbai, India. ${ }^{3}$ University of Southern California, Los Angeles, CA, USA.

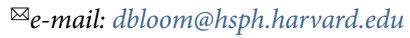

Published online: 30 November 2021

https://doi.org/10.1038/s43587-021-00155-y

\section{References}

1. United Nations, Department of Economic and Social Affairs, Population Division. World Population Prospects 2019, Online Edition, Rev. 1, https://go.nature.com/3cFqF0R (2019).

2. Giridhar, G., Sathyanarayana, K. M. \& James, K. S. in Population Ageing in India (eds. Giridhar, G. et al.) xvii-xxvi (Cambridge Univ. Press, 2014)

3. World Health Organization. Decade of Healthy Aging: Plan of Action, https://go.nature.com/3HjQOQS (14 December 2020).
4. International Institute for Population Sciences (IIPS) et al. Longitudinal Ageing Study in India (LASI) Wave 1, 2017-18, India Report, https://go.nature.com/2YOoDbk (2020).

5. University of Michigan. Health and Retirement Study, https:// go.nature.com/3FfxeTW (2017).

6. Zhao, Y. et al. China Health and Retirement Longitudinal Study Wave 4 User's Guide (National School of Development, Peking Univ., 2020).

7. Lee, J. et al. J. Am. Geriatr. Soc. 68 (Suppl 3), S5-S10 (2020).

8. Bloom, D. E. et al. (2021). Addressing Alzheimer's disease and related dementias to realise the promise of the UN's 'Decade of Healthy Ageing. voxeu.org, https://go.nature.com/3Dqkdqf (26 March 2021).

9. Jia, J. et al. Alzheimers Dement. 14, 483-491 (2018).

10. Rashid, M., Muhammad, T. \& Srivastava, S. Geriatr. Nurs. 42, 1446-1453 (2021)

11. Ehrlich, J. R., Ndukwe, T., Chien, S. \& Lee, J. Neuroepidemiology 55, 126-134 (2021).

12. Angrisani, M., Jain, U. \& Lee, J. J. Am. Geriatr. Soc. 68(Suppl 3), S20-S28 (2020).

13. Mohanty, S. K. et al. PLoS Med. 18, e1003740 (2021).

14. Lee, J. et al. PLoS Med. (in the press).

15. Jin, H., Chien, S., Meijer, E., Khobragade, P. \& Lee, J. JMIR Ment. Health 8, e27113 (2021).

16. Lee, J. \& Dey, A. B. J. Am. Geriatr. Soc. 68(Suppl 3), S3-S4 (2020).

\section{Acknowledgements}

This project is funded by the National Institute on Aging, National Institutes of Health, the USA (R01 AG042778, 2R01 AG051125 and U01AG065958), and the Ministry of Health and Family Welfare, Government of India (T22011/02/2015-NCD). The authors thank J. Adams and A. Agarwal for helpful editorial and research assistance. The authors would also like to acknowledge P. Arokiasamy's role and contribution as one of the original co-PIs of LASI, from its inception through his retirement in August 2020.

Author contributions

All authors contributed to the conception, drafting and revision of the article.

Competing interests

The authors declare no competing interests. 